\title{
Well-being in the Intensive Care Unit
}

\section{Looking Beyond COVID-19}

Sheela Pai Cole, MD ${ }^{a, *}$, Shahla Siddiqui, MD, DABA, MSc, FCCM ${ }^{b}$

\section{KEYWORDS}

- Compassion fatigue - Burnout syndrome - Compassion satisfaction

- Secondary traumatic stress $\bullet$ Moral injury • Psychological resilience

\section{KEY POINTS}

- Wellbeing in the intensive care unit (ICU) is affected by a combination of personal factors, organizational factors, quality of interpersonal relationships, and exposure to end-of-life issues.

- Moral injury occurs when an act is perpetrated, one bears witness to or fails to prevent an act that is against deeply held moral beliefs.

- Second victim syndrome is the guilt and other psychological onslaught faced by health care providers who hold themselves responsible following unexpected patient morbidity or mortality.

- Leadership held check-ins, active listening to feedback, and availability of wellness resources help mitigate health care worker (HCW) burnout.

- Training in communication, conflict resolution, and simulation of team-based care aid in creating collaborative scenarios and clarifying roles among multi-disciplinary teams.

\section{INTRODUCTION}

Critical care medicine is a subspecialty characterized by intense clinical situations balanced by immense reward in the care of critically ill patients in the intensive care unit (ICU). ${ }^{1,2}$ Intensivists often work in multidisciplinary paradigms, frequently engaging with other physicians, advanced practice providers, nurses, and ancillary

\footnotetext{
${ }^{a}$ Department of Anesthesiology, Perioperative and Pain Medicine, Stanford University, 300 Pasteur Drive, H3580, Stanford, CA 94305, USA; ${ }^{b}$ Department of Anesthesia, Critical Care and Pain Medicine, Beth Israel Deaconess Medical Center, Harvard Medical School, 330 Brookline Avenue, Boston, MA 02215, USA

* Corresponding author.

E-mail address: spaicole@stanford.edu

Twitter: @SheelaPaiCole (S.P.C.); @shahlasi (S.S.)
} 
staff. ${ }^{3,4}$ The critical care team works in tandem to manage complicated, lifethreatening illnesses, initiate end-of-life conversations, collaborate with consultants, among many other high-acuity tasks. ${ }^{4}$ These cumulative demands may, over time, contribute to an intensivist's perceived lack of autonomy and poor work-life balance. ${ }^{5,6}$ Coupled with frequent sleep deprivation, this may result in an increased incidence of depression, anxiety, and burnout syndrome (BOS) in ICU physicians compared with other physicians. ${ }^{7}$ Burnout is more common among physicians compared with the general population. Global literature has shown a high rate of burnout within all specialties of medicine. ${ }^{2}$ The 2020 National Physician Burnout and Suicide Report showed a $44 \%$ rate of burnout among ICU physicians compared with $41 \%$ of general anesthesiologists. ${ }^{8,9}$ The above description has been amplified by the ongoing COVID-19 pandemic not only due to dying patients but also personal factors such as changes in workload and wages coupled with job insecurity. ${ }^{10,11}$ There have been a multitude of metaphorical images depicting the "physician as hero" role, such as the submerged physician holding a patient bed afloat -in other words, saving the patient at the cost of drowning themselves.

Considering the existence of widespread incidence of BOS among all levels of ICU practitioners before the COVID-19 pandemic, in 2016 an official statement entitled "Burnout Syndrome in Critical Care Health care Professionals. A Call for Action," was jointly prepared and published by the American Thoracic Society (ATS), American College of Chest Physicians (CHEST), American Association of Critical-Care Nurses (AACN), and Society of Critical Care Medicine (SCCM) in their corresponding professional journals. ${ }^{12}$ Although the statement predates the pandemic, many of the factors predisposing to BOS highlighted in the report are relevant and magnified in the current moment, such as at-risk personal characteristics, work-life balance, and organizational pressures. These factors may cumulatively lead to a high rate of turnover, poor quality of patient care, and reduced patient satisfaction. Another initiative in 2020 was the Critical Care Societies' collaborative National Summit and Survey on Prevention and Management of Burnout in the ICU. The summit identified that society is at risk of losing this essential workforce and it is imperative to investigate the root causes of ICU clinician BOS. Addressing physician and caregiver well-being is important for staff retention and is associated with improved patient outcomes, increased patient satisfaction, lower infection rates, and lower medication errors. ${ }^{13}$

\section{Wellbeing Versus Burnout Syndrome}

Well-being in the workplace is defined as "the presence of professional fulfillment and the absence of burnout." 14 Stamm and colleagues, coined the term Professional Quality-of-life (ProQOL) which consists of both positive and negative elements (ie, compassion satisfaction and compassion fatigue are important determinants of well-being in the workplace). ${ }^{15,16}$ Maslach and colleagues, defined the term BOS by assessing 3 components: (1) emotional exhaustion, (2) depersonalization, and (3) lack of professional achievement or fulfillment. ${ }^{17}$ The efficacy of validated instruments to assess burnout such as ProQOL and BOS increases when results are garnered from groups working in similar environments. ${ }^{15}$

Instruments for data collection such as Professional Quality of Life (ProQOL) have been used in various health care communities to demonstrate a direct association between the presence of compassion fatigue (ie, the negative feeling associated with constant self-giving leading to discomfort) and an increased incidence of BOS as well as an inverse relationship between compassion satisfaction (ie, the positive feeling associated with helping others and often the premise that leads health care workers (HCWs) to their careers) and BOS. ${ }^{18}$ Compassion fatigue and BOS are 
more commonly seen among ICU nurses compared with ICU physicians, which might be attributed to the current crisis of nation-wide nursing shortages.

\section{Concepts and Definitions Associated with Well-being Literature}

Compassionate care is essential to health care delivery. Compassion satisfaction, compassion fatigue, burnout, and secondary traumatic stress are all frequently coined terms when discussing the correlates of total well-being. It is equally important to have the definitions imprinted as it is to have real-world examples to reflect on. Compassion satisfaction is the positive feeling associated with helping others. It is the passion that drives most physicians to start careers in medicine. It is the satisfaction an ICU physician experiences when a patient previously on life-support returns to the ICU for a visit after being discharged home. This is in direct contrast to compassion fatigue. Compassion fatigue was initially described by Figley in 1982 as "the cost of caring," which is amplified when health care workers are unable to refuel. ${ }^{19,20}$ It describes the emotional toll that occurs with a continued outpouring of compassionate care. It is frequently seen among health care providers who seem unfazed by death and dying. BOS is the individual response to work-related events that occur in workers without baseline psychological disorders. A diagnosis of BOS requires all 3 components, as described by Christina Maslach: high depersonalization, high emotional exhaustion, and low personal fulfillment. Finally, secondary traumatic stress is the stress experienced by witnesses to trauma, usually as a "bystander." 21,22 This is especially true among ICU nurses who are frequently not decision makers but partake in the dayto-day care of the ailing patient.

\section{Implications of the Coronavirus Pandemic}

The COVID-19 pandemic has exacted enormous health care, economic, and psychological burdens globally. ${ }^{23}$ Devastating effects of the pandemic include: high rates of infection and death, financial hardships faced by society and individuals, anxiety due to uncertainty of the future, personal health-related outcomes, and stress of childcare, to name a few. ${ }^{24} \mathrm{HCW}$ are at the frontlines of the pandemic and face ongoing unprecedented challenges in treating unmanageable surges of patients with COVID-19, in working with public health officials to decrease the spread of infection, developing suitable short-term strategies, and formulating long-term plans. ${ }^{25}$ The World Health Organization (WHO) estimates approximately 179,500 HCWs have died due to the COVID-19 pandemic worldwide. ${ }^{26}$ The psychological burden and overall wellness of HCWs, and intensivists in particular, has received increased awareness, as the lay press and medical literature continue to report high rates of burnout, psychological stress, and suicide among clinicians including ICU providers. ${ }^{27,28}$ Reports of increased COVID-19-related stress, anxiety, depression, burnout, suicide, and PTSD among frontline ICU staff including physicians are increasing. ${ }^{28}$

The high rate of COVID-19 mortality compounded by ongoing surges due to inconsistent vaccination in the US and globally and the politicization of COVID-19 therapies and inoculation has contributed to alarming rates of emotional exhaustion and burnout among HCWs, many of whom are now leaving the profession. ${ }^{20}$ Additional challenges faced by intensivists include: fear of infecting family and loved ones, job insecurity due to potentially protracted personal illness, anxiety regarding the supply and quality of personal protective equipment (PPE), adapting to ever-changing hospital guidelines and operating procedures and policies, sustaining physically demanding and long work hours, particularly when faced with staffing shortages, and reconciling a professional commitment to helping others with the need to protect one's self. ${ }^{18}$ 


\section{Risk Factors for Burnout among Intensive Care Unit Personnel}

BOS risk factors can be grouped into 4 main categories. ${ }^{12}$ Fessell and colleagues suggest a fifth category described later in discussion as a recent addition based on lessons learned from caring for patients during the pandemic. ${ }^{25}$

1. Personal characteristics: self-criticism, idealism, perfectionism, inadequate coping strategies, sleep deprivation, and overcommitment. These characteristics are often seen among highly productive workers. ${ }^{21}$

2. Organizational factors: unmanageable workload, lack of control over the work environment, insufficient rewards, and general breakdown in the work community. Specifically, for critical care physicians, night shifts and lack of time off between ICU weeks increased burnout, whereas for nurses, lack of ability in choosing days off and rapid patient turnover increased BOS. ${ }^{23}$

3. Quality of working relationships is an important modifiable risk factor for BOS. The conflicts between members of multidisciplinary teams caring for a complex patient as well as difficult HCW-patient relationships exact a toll on clinician well-being.

4. Exposure to end-of-life issues: Critical Care nurses cite increased burnout related to care of a dying patient-witnessing, and participating in transitions to comfort care Critical care physicians cite increased burnout due to: the constant exposure to inappropriate care (this can be related to delays in care and/or the wrong amount of care) (an example would include delivery of inaccurate information to a patient or family, disrespecting a patient's wishes, and advocating that another patient might benefit from an ICU bed as issues that compound). ${ }^{24,29}$

5. The pandemic has unearthed unprecedented anxiety and conflict among HCWs due to resource shortages and politicization of standard preventative measures such as wearing a mask, social distancing, avoiding super-spreader events, and vaccination to protect against severe disease. This is magnified among ICU personnel as they care for the most critically ill patients that often require resource-intensive advanced therapies including mechanical ventilation and extracorporeal membrane oxygenation. ${ }^{25}$

\section{Moral Injury and Intensive Care Unit Practice}

Moral injury occurs when an act is perpetrated, one bears witness to or fails to prevent an act that is against deeply held moral beliefs. ${ }^{30}$ The term was first used in health care to describe the emotional turmoil suffered by nursing personnel after long hours of patient care that ultimately resulted in an unavoidable fatal outcome. ${ }^{22}$ Among health care providers, any act that is in contradiction to the Hippocratic oath or serves as an impediment to deliver safe care to patients may be a source of moral injury. Intensivists may face several triggers for moral injury, such as observing undue patient suffering from delays in end-of-life discussions or poor clinical decision-making, communication challenges in brain death notifications, misunderstandings regarding do not resuscitate status, inappropriate or inaccessible care delivery, poor outcomes due to health delivery disparities, psychiatric issues and addiction leading to suboptimal outcomes, and engaging with grieving families. ${ }^{31}$ Critical care physicians are often drawn to the field to care for the seriously ill; however, complex regulatory, insurance, and quality reporting requirements have led to increased documentation and other administrative activities, drawing away from time spent that would otherwise be spent at the bedside. This incongruence between clinician ideals and the reality of clinical medicine may further contribute to feelings of dissatisfaction. ${ }^{3}$

The COVID-19 pandemic has wrought additional moral injury, as intensivists continue to manage unprecedented volumes of high-acuity patients and navigate 
unknown and often futile treatment options. ${ }^{28}$ The moral, psychological, and physical exhaustion and injury adds to compassion fatigue, medical errors, a lack of empathy in treating patients and caring for families, lower productivity, and higher staff turnover rates. The ability of HCWs to adequately cope with these stressors is important for their patients, their families, and for themselves. Left unchecked, long term, severe stressors can contribute to significant physical and mental health problems and low "psychological resilience" (the ability to positively adapt to adversity to protect themselves from stress). Another pandemic-induced burden to critical care personnel is the ethical constraints of shortages of ICU beds, ventilators, essential medicines, PPE, and staffing. Critical care personnel are tasked with enormously distressing difficult decisions around resource allocation, triaging patients, and assigning extremely limited resources that are essential to save a patient's life. ${ }^{32}$ This further compounds moral burden and distress. ${ }^{19}$

\section{Second Victim Syndrome}

Second victim syndrome is defined as the guilt and psychological onslaught faced by health care providers who feel responsible following an unexpected patient morbidity or mortality event. ${ }^{33}$ It may also refer to the struggle faced by HCWs from medical errors, unmanageably long working hours, under-supported medical practices, and lack of psychological support after a patient fatality or major adverse event. ${ }^{34,35}$ In addition to the primary caregiver, second victims may include colleagues called in to help during an acute event, support personnel, students, and others who may have been involved in the event or the immediate aftermath. ${ }^{36}$

Second victims often experience fear, guilt, self-doubt, shame, anger, reliving of the event, sleep disturbance, and anxiety. ${ }^{29,37}$ These emotions mirror symptoms of acute stress disorder and posttraumatic stress disorder and may affect HCWs both at work and at home. Such emotions may persist for weeks to years and lead to burnout, substance abuse, and even suicide. In a 2012 national survey of the impact of perioperative catastrophes on anesthesiologists, more than $60 \%$ of respondents experienced depression and $19 \%$ indicated that they never fully recovered from the experience and felt that their ability to provide care for the first 4 hours after an adverse event was impaired. ${ }^{37}$ Although there is a dearth of literature among ICU physicians, the rate of second victim syndrome has been shown to be anywhere from $10 \%$ to $40 \%$ in this group. ${ }^{35}$

\section{Solutions to Mitigate Burnout and Improve Intensivist Fulfillment}

Later in discussion, we describe pandemic-specific measures to address well-being and discuss long-term solutions to mitigate BOS and improve overall well-being in the critical care community.

\section{COVID-19-SPECIFIC MEASURES TO IMPROVE CRITICAL CARE CLINICIAN WELL-BEING Community Building}

The pandemic reified the importance of a workplace community in mitigating burnout and promoting well-being. Clinical leaders can build and reinforce resilience in their teams by displaying compassion. In the authors' collective experience, we have witnessed thoughtful leaders checking in frequently, listening to feedback, providing wellness resources and support, and demonstrating compassion to their team. ${ }^{38}$ This sense of togetherness and care can significantly bolster team morale and sustain clinicians during highly stressful times. Guided conversations or town halls to foster communication can provide reassurance of future stability and help staff collectively 
envision a future beyond the pandemic. 39 "Work-from-home regime engagement activities" are useful for employees as well as for institutions. "Those organizations doing these kinds of engagement activities for their employees are learning new skills and developing themselves." 40 This hopeful outlook begins with leadership and serves as a sustaining source of team morale. Indeed, in times of extreme stress, teams need to trust and rely on one another and leadership for support. ${ }^{41-43}$

\section{Long-term Measures to Mitigate Burnout}

\section{Communication training}

Institutional commitment to mitigate intensivist BOS could include the incorporation of team building and community building programs that strengthen team dynamics and patient care. Incorporating daily multidisciplinary rounds (MDR) helps to clarify roles among various ICU providers including nursing, respiratory therapy, physical and occupational therapy, and social workers. MDRs also aid in the synthesis of comprehensive care plans for patients and facilitate ICU discharge. ${ }^{44}$ In their postimplementation survey, O'Brien and colleagues, demonstrated the feasibility of incorporating MDR into the ICU workflow and improved overall communication between all members of the ICU team. ${ }^{44}$ Debriefings after adverse events such as cardiac arrest are another example of fostering team building. Dedicated time for debriefing lauds effective team actions identifies opportunities for future growth and create collaborative scenarios between teams. ${ }^{45,46}$ In a single-center postcode debriefing survey, code response satisfaction improved ${ }^{47}$ and staff members described an increase in peer and institutional support after the implementation of a debriefing protocol. ${ }^{48}$

Further, dedicated training in communication and conflict resolution via team-based care simulation may be helpful. For example, Vitaltalk, a team-based simulation training program, is being adopted at many institutions to hone critical care trainees' communication skillsets to bridge patient values with treatment plans. ${ }^{49}$ These programs intend to reduce BOS among ICU staff and hasten emotional healing after stressful events.

\section{Post-intensive Care Unit Clinics and Longevity Programs}

Post-ICU clinics help patients and their families re-acclimatize to normal life and allow ICU providers to experience post-ICU outcomes. Sharing details of patient survival constitutes continuity of care and serves as a form of evaluation and validation for the care provided during the ICU course and may boost clinician morale. ${ }^{42}$ As an example, The Society of Critical Care Medicine's (SCCM) THRIVE program provides resources and education for ICU patient survivors and their families related to postintensive care syndrome and has had a significant impact on ICU physician morale and well-being. ${ }^{42}$ Other benefits of post-ICU programs include identifying unforeseen outcomes of ICU therapies (eg, stop dates for certain medications), forming a network of ICU survivors as support for other critically ill patients and families, facilitating postICU follow-up education to ICU providers, and providing insight into the patient experience during an ICU stay. One challenge of sustaining a post-ICU program is that fiscal support typically falls outside traditional payment models and requires sustainable institutional investment.

\section{Flexible Scheduling}

Control of one's schedule is an important determinant of personal and professional satisfaction. With the advent of high acuity intensive care units (ICU) caring for patients on mechanical circulatory support, large-volume centers are attempting to move toward continuous in-house coverage by critical care faculty. Purported advantages 
of this model are improved patient care and patient satisfaction, especially when this care model is accompanied by a "system change." System change involves the institution of measures such as liberation from mechanical ventilation, goals of care conversations, and other interventions around the clock instead of deferring these "nonemergent" tasks to the day team caring for the patient. ${ }^{41,43,50}$

Traditional ICU coverage is allocated in 7-day assignments with integrated overnight coverage. This model can result in physicians spending more than 33 consecutive hours in the ICU. Proponents of the traditional model tout continuity of care and patient safety as the biggest advantages of this approach. Some institutions have mitigated long ICU shifts by creating a shift-based system (ie, separate day and night shifts). Concerns with shift-based models include discontinuity of care and patient safety as there are more frequent hand-offs between ICU personnel and greater coverage of nights and weekends throughout the year. Geva and colleagues, recently described a simulated model of shared service scheduling whereby 4 ICU attendings shared most of the day and nighttime service for 2 teams over a 2-week period with creative assignments to avoid 30 plus hour shifts. ${ }^{51}$ This simulation study found more continuity of care and less handoffs, making it safer for patients while facilitating improved intensivist work-life balance with more weekends off throughout the year. Further, implementation data on this simulation paradigm are awaited.

\section{SUMMARY}

Advocating for personal well-being strategies such as self-care, self-forgiveness, and mindfulness may anecdotally help with reducing burnout and increasing professional well-being. However, institutional support in incorporating efficient practices and building a culture of wellness through systems-based changes may be more effective in recruiting and retaining a well workforce. ${ }^{3,52,53}$ Appointing institutional or departmental well-being officers who are well versed in clinical workflow inefficiencies and have the skillset to advocate for their peers and colleagues are essential in supporting an institutional commitment to clinician well-being. ${ }^{11}$ Finally, the COVID-19 pandemic has created a unique psychological quagmire among critical care professionals. Reducing intensivist burnout through proactive, multifaceted measures by institutions can help retain motivated and patient-centered clinicians that can continue providing the quality of care that we envision for our own loved ones.

\section{DISCLOSURE}

The authors have nothing to disclose.

\section{CLINICS CARE POINTS}

- Moral injury occurs when an act is perpetrated, one bears witness to or fails to prevent an act that is against deeply held moral beliefs, especially in the care of a patient at the end of their lives. Having multidisciplinary debriefings to discuss different viewpoints among the various caregivers may help the team to understand the varying opinions and basis of care being provided.

- Lack of control over one's control schedule can be addressed by incorporating flexible scheduling paradigms.

- Difficult relationships among members of different specialties caring for the same critically ill patient can be modified by providing communication and conflict resolution training, and simulation programs for team-based care 
- Understanding a patient's progress after leaving the ICU by investing in post-ICU clinics helps HCWs realize the value of their efforts as well as gain perspective on the consequences of ICU therapies.

\section{REFERENCES}

1. Shanafelt T, Goh J, Sinsky C. The Business Case for Investing in Physician Wellbeing. Jama Intern Med 2017;177(12):1826.

2. Shanafelt TD. Enhancing Meaning in Work: A Prescription for Preventing Physician Burnout and Promoting Patient-Centered Care. Jama 2009;302(12): 1338-40.

3. Shanafelt T, Trockel M, Ripp J, et al. Building a Program on Well-Being: Key Design Considerations to Meet the Unique Needs of Each Organization. Acad Med J Assoc Am Med Coll 2019;94(2):156-61.

4. Cole SP. Burnout Prevention and Resilience Training for Critical Care Trainees. Int Anesthesiol Clin 2019;57(2):118-31.

5. Schäfer SK, Sopp MR, Staginnus M, et al. Correlates of mental health in occupations at risk for traumatization: a cross-sectional study. Bmc Psychiatry 2020; 20(1):335.

6. Geronazzo-Alman L, Eisenberg R, Shen S, et al. Cumulative exposure to workrelated traumatic events and current post-traumatic stress disorder in New York City's first responders. Compr Psychiat 2017;74:134-43.

7. Mealer M. Burnout Syndrome in the Intensive Care Unit. Future Directions for Research. Ann Am Thorac Soc 2016;13(7):997-8.

8. Pines A, Maslach C. Characteristics of Staff Burnout in Mental Health Settings. Psychiatr Serv 1978;29(4):233-7.

9. Medscape National Physician Burnout \& Suicide Report 2020: The Generational Divide.pdf. Available at: https://www.medscape.com/slideshow/2020-lifestyleburnout-6012460. Accessed December 5, 2021.

10. Mitchell EP. Clinician Wellbeing during COVID-19. J Natl Med Assoc 2021; 113(5):481.

11. Dzau VJ, Kirsch D, Nasca T. Preventing a Parallel Pandemic - A National Strategy to Protect Clinicians' Well-Being. N Engl J Med 2020. https://doi.org/10.1056/ nejmp2011027.

12. Moss M, Good VS, Gozal D, et al. A Critical Care Societies Collaborative Statement: Burnout Syndrome in Critical Care Health-care Professionals. A Call for Action. Am J Resp Crit Care 2016;194(1):106-13.

13. Kleinpell R, Moss M, Good VS, et al. The Critical Nature of Addressing Burnout Prevention: Results From the Critical Care Societies Collaborative's National Summit and Survey on Prevention and Management of Burnout in the ICU. Crit Care Med 2020;48(2):249-53.

14. Stobbs C. Maintaining personal resilience in this Covid-19 era. Practice 2021; 43(2): 109-12.

15. Stamm Beth. ProQOL Manual.pdf. https://img1.wsimg.com/blobby/go/dfc1e1a0a1db-4456-9391-18746725179b/downloads/ProQOL\%20Manual.pdf? ver $=1622839353725$.

16. Xiong J, Lipsitz O, Nasri F, et al. Impact of COVID-19 Pandemic on Mental Health in the General Population: A Systematic Review. J Affect Disord 2020;277:55-64.

17. Lahav Y. Psychological distress related to COVID-19 - The contribution of continuous traumatic stress. J Affect Disord 2020;277:129-37. 
18. Sterling MR, Tseng E, Poon A, et al. Experiences of Home Health Care Workers in New York City During the Coronavirus Disease 2019 Pandemic. Jama Intern Med 2020;180(11):1453-9.

19. Lai J, Ma S, Wang Y, et al. Factors Associated With Mental Health Outcomes Among Health Care Workers Exposed to Coronavirus Disease 2019. Jama Netw Open 2020;3(3):e203976.

20. Shen X, Zou X, Zhong X, et al. Psychological stress of ICU nurses in the time of COVID-19. Crit Care 2020;24(1):200.

21. Poncet MC, Toullic P, Papazian L, et al. Burnout Syndrome in Critical Care Nursing Staff. Am J Resp Crit Care 2012;175(7):698-704.

22. Jameton A. What Moral Distress in Nursing History Could Suggest about the Future of Health Care. Ama J Ethics 2017;19(6):617-28.

23. Embriaco N, Azoulay E, Barrau K, et al. High Level of Burnout in Intensivists. Am J Resp Crit Care 2007;175(7):686-92.

24. Piers RD, Azoulay E, Ricou B, et al. Perceptions of Appropriateness of Care Among European and Israeli Intensive Care Unit Nurses and Physicians. Jama 2011;306(24):2694-703.

25. Fessell D, Cherniss C. COVID-19 \& Beyond: Micro-practices for Burnout Prevention and Emotional Wellness. J Am Coll Radiol 2020;17(6):746-8.

26. Organization $\mathrm{WH}$, department $\mathrm{H}$ workforce. The Impact of COVID-19 on health and care workers: a closer look at deaths.

27. Chew NWS, Lee GKH, Tan BYQ, et al. A multinational, multicentre study on the psychological outcomes and associated physical symptoms amongst healthcare workers during COVID-19 outbreak. Brain Behav Immun 2020;88:559-65.

28. Restauri N, Sheridan AD. Burnout and Posttraumatic Stress Disorder in the Coronavirus Disease 2019 (COVID-19) Pandemic: Intersection, Impact, and Interventions. J Am Coll Radiol 2020;17(7):921-6.

29. Seys D, Wu AW, Gerven EV, et al. Health Care Professionals as Second Victims after Adverse Events. Eval Health Prof 2013;36(2):135-62.

30. Dean W, Talbot S, Dean A. Reframing Clinician Distress: Moral Injury Not Burnout. Fed Pract Heal Care Prof Va Dod Phs 2019;36(9):400-2.

31. Rosenthal MS, Clay M. Initiatives for Responding to Medical Trainees' Moral Distress about End-of-Life Cases. Ama J Ethics 2017;19(6):585-94.

32. Kenny N, Kotalik J, Herx L, et al. A Catholic Perspective: Triage Principles and Moral Distress in Pandemic Scarcity. Linacre Q 2021;88(2):214-23.

33. Wu AW. Medical error: the second victim. The doctor who makes the mistake needs help too. Bmj Clin Res Ed 2000;320(7237):726-7.

34. Busch IM, Scott SD, Connors C, et al. The Role of Institution-Based Peer Support for Health Care Workers Emotionally Affected by Workplace Violence. Jt Comm J Qual Patient Saf 2021;47(3):146-56.

35. Ozeke O, Ozeke V, Coskun O, et al. Second victims in health care: current perspectives</p\&gt. Adv Med Educ Pract 2019;10:593-603.

36. Scott SD, Hirschinger LE, Cox KR, et al. The natural history of recovery for the healthcare provider "second victim" after adverse patient events. Qual Saf Heal Care 2009;18(5):325-30.

37. Gazoni FM, Amato PE, Malik ZM, et al. The Impact of Perioperative Catastrophes on Anesthesiologists. Anesth Analg 2012;114(3):596-603.

38. Giordano F, Cipolla A, Ungar M. Building resilience for healthcare professionals working in an Italian red zone during the COVID-19 outbreak: A pilot study. Stress Health 2021. https://doi.org/10.1002/smi.3085. 
39. Mosanya M. Buffering Academic Stress during the COVID-19 Pandemic Related Social Isolation: Grit and Growth Mindset as Protective Factors against the Impact of Loneliness. Int J Appl Posit Psychol 2020;6(2):159-74.

40. Chanana N, Sangeeta. Employee engagement practices during COVID-19 lockdown. J Public Aff 2020;e2508.

41. Mikkelsen ME, Anderson BJ, Bellini L, et al. Burnout, and Fulfillment, in the Profession of Critical Care Medicine. Am J Resp Crit Care 2019;200(7):931-3.

42. Haines KJ, Sevin CM, Hibbert E, et al. Key mechanisms by which post-ICU activities can improve in-ICU care: results of the international THRIVE collaboratives. Intensive Care Med 2019;45(7):939-47.

43. Kerlin MP, McPeake J, Mikkelsen ME. Burnout and Joy in the Profession of Critical Care Medicine. Crit Care 2020;24(1):98.

44. O'Brien A, O'Reilly K, Dechen T, et al. Redesigning Rounds in the ICU: Standardizing Key Elements Improves Interdisciplinary Communication. Jt Comm J Qual Patient Saf 2018;44(10):590-8.

45. Couper K, Kimani PK, Davies RP, et al. An evaluation of three methods of inhospital cardiac arrest educational debriefing: The cardiopulmonary resuscitation debriefing study. Resuscitation 2016;105:130-7.

46. Dine CJ, Gersh RE, Leary M, et al. Improving cardiopulmonary resuscitation quality and resuscitation training by combining audiovisual feedback and debriefing\&ast. Crit Care Med 2008;36(10):2817-22.

47. Przednowek T, Stacey C, Baird K, et al. Implementation of a Rapid Post-Code Debrief Quality Improvement Project in a Community Emergency Department Setting. Spartan Med Res J 2021;6(1):21376.

48. Copeland D, Liska H. Implementation of a Post-Code Pause. J Trauma Nurs 2016;23(2):58-64.

49. Markin A, Cabrera-Fernandez DF, Bajoka RM, et al. Impact of a Simulation-Based Communication Workshop on Resident Preparedness for End-of-Life Communication in the Intensive Care Unit. Crit Care Res Pract 2015;2015:534879.

50. Nizamuddin J, Tung A. Intensivist staffing and outcome in the ICU. Curr Opin Anaesthesiol 2019;32(2):123-8.

51. Geva A, Landrigan CP, van der Velden MG, et al. Simulation of a Novel Schedule for Intensivist Staffing to Improve Continuity of Patient Care and Reduce Physician Burnout. Crit Care Med 2017;45(7):1138-44.

52. Shanafelt T, Swensen S. Leadership and Physician Burnout: Using the Annual Review to Reduce Burnout and Promote Engagement. Am J Med Qual 2017;32(5): 563-5.

53. Shanafelt T, Ripp J, Trockel M. Understanding and Addressing Sources of Anxiety Among Health Care Professionals During the COVID-19 Pandemic. Jama 2020;323(21):2133. 